Case Report

\title{
Novel TRAPPC11 Mutations in a Chinese Pedigree of Limb Girdle Muscular Dystrophy
}

\author{
Xike Wang $\mathbb{D}^{1},{ }^{1}$ Yue Wu, ${ }^{1}$ Yuxia Cui, ${ }^{1}$ Nan Wang, \\ Lasse Folkersen $\mathbb{D}^{2},{ }^{2}$ and Yuchuan Wang $\mathbb{D}^{1}$ \\ ${ }^{1}$ Department of Pediatrics, Guizhou Provincial People’s Hospital, Guiyang, Guizhou 559992, China \\ ${ }^{2}$ Sankt Hans Hospital, Capital Region Denmark, Roskilde 4000, Denmark \\ Correspondence should be addressed to Xike Wang; wangxike2008@sina.com
}

Received 25 October 2017; Accepted 22 May 2018; Published 16 July 2018

Academic Editor: Mohnish Suri

Copyright (C) 2018 Xike Wang et al. This is an open access article distributed under the Creative Commons Attribution License, which permits unrestricted use, distribution, and reproduction in any medium, provided the original work is properly cited.

Limb girdle muscular dystrophies (LGMDs) are a heterogeneous group of genetic myopathies leading primarily to proximal muscle weakness. It is caused by mutations at over 50 known genetic loci typically from mutations in genes encoding constituents of the sarcolemmal dystrophin complex or related functions. Herein we describe the case of two siblings with LGMD that were investigated using whole-exome sequencing followed by Sanger sequencing validation of a specific double-mutation in the TRAPPC11 gene. Further, from parental sequencing we determined the mode of transmission, a double heterozygous mutation at the maternal and paternal alleles. The two mutations detected have not been described in other patients.

\section{Introduction}

More than 50 mutations have been described for LGMD, all having either autosomal-dominant (termed LGMD1) or autosomal-recessive inheritance (termed LGMD2) [14]. As such, LGMD constitutes a heterogeneous group of myopathies, but all leading to proximal muscle weakness, with relative sparing of heart and bulbar muscles. The major forms of LGMD result from mutations in genes encoding constituents of the sarcolemmal dystrophin complex, e.g., laminin $(L G M D 1 B)$, sarcoglycan (LGMD2C-F), and dysferlin $(L G M D 2 B)$. Other forms have also been described, typically resulting from mutations in genes affecting muscle function involving membrane trafficking [5], muscle remodeling [6], and posttranslational modification of sarcolemmal proteins [7]. The age of onset, severity, and rate of progression vary considerably between LGMD subtypes, ranging from early childhood myopathy to adult onset with long-time preserved ambulation. Here, we reported the clinical and molecular phenotype of an autosomal-recessive form of LGMD caused by two novel TRAPPC11 mutations in a Chinese family of Buyi origin with two affected members.

\section{Case Presentation}

The first affected individual was from a Chinese family of Buyi origin, a girl born to healthy unrelated parents after an uneventful pregnancy. The affected individual presented at age 5 with a progressive proximal muscle weakness and difficulty in standing up from sitting and walking, particularly stair-climbing. This started at approximately age 2 and gradually worsened. Physical examination was unremarkable except for mild short stature. No other muscle groups, including upper limbs, were not affected. No cataracts were observed. Echocardiogram and abdominal ultrasound scan was likewise unremarkable.

The second affected individual was a younger boy, born to the same parents. He presented at the same time, at age 3 , with a similarly progressive proximal muscle weakness and the same developmental history as the first individual. Physical examination was likewise unremarkable, with no other muscle groups, affected, no remarks in ultrasound, and no cataracts.

In both individuals, there were no significant signs of extramuscular involvement. Biochemistry analysis revealed more than 100-fold increase of serum creatine kinase 
(CK) levels, markedly elevated serum lactate dehydrogenase level (1747 U/L) and $\alpha$-Hydroxybutyrate dehydrogenase (1237 U/L) and mildly elevated serum glutamic-oxaloacetic transaminase (142U/L). The electromyogram (EMG) examination showed a typical muscle-derived damage in both affected children. No MRI or muscle biopsies were performed.

2.1. Whole-Exome Sequencing. Genomic DNA samples were extracted with Gentra Puregene Kit (Qiagen, Germany) from peripheral blood collected from the patient and his parent. The quality and quantity of genomic DNA sample were determined using a spectrophotometer (NanoDrop, USA). Genomic DNA library was prepared with Agilent SureSelect Human All Exon Kit v5 reagents, as instructed by the manufacturer's standard protocol. The enriched DNA samples were sequenced with Hiseq2000 instrument (Illumina) using $2 \times 100$ paired-end sequencing. The Illumina Sequencing Control Software (SCS) v2.8, the Illumina Off-Line Basecaller Software (OLB) v1.8, and the Illumina Consensus Assessment of Sequence And VAriation (CASAVA) v1.8 were used to produce $100 \mathrm{bp}$ sequence reads. The study was approved by the Ethics Committee of People's Hospital of Guizhou Province, and written informed consent was obtained from their parents of the two affected individuals.

\subsection{Alignment, Variant Calling, and Annotation. Burrows-} Wheeler Aligner (BWA) [8] was used to align sequence reads to the human reference genome (hg19) with default parameters and variants were called using the Genome Analysis Toolkit (GATK) software package VarScan [911]. Coverage was determined using the CalculateHsMetrics mode of Picard software. The following analytical steps were performed only with reads that matched exonic regions including exon-intron-boundaries. SNP and insertion/deletion (indels) analysis was done by different filtering steps. The resulting list of variants was annotated with Annovar [12] that summarizes and utilizes information from external databases to assess implications and consequences of a given sequence alteration, such as amino acid change, location within a canonical splice site, and information from dbSNP along with the SNP frequency if available. Finally, a manual filtering step was carried out to prioritize relevant genes in the 30 major LGMD genes for $L G M D 1 B, L G M D 2 C$ $F$, and $L G M D 2 B$.

2.3. Variants Filtering. The variant detection frequency was set with a threshold for variant consideration at a minimum of $20 \%$ of the reads and an absolute read minimum coverage of 10 reads. In each case all variants listed in the most recent version of the NCBI (National Center for Biotechnology Information) dbSNP database were excluded as well as silent mutations. Low frequency frameshift and truncating mutations in any LGMD gene were considered pathogenic. Unreported nonsynonymous amino acid variants were analyzed by MutationTaster (http://www.mutationtaster.org), Polyphen-2 (http://genetics.bwh.harvard.edu/pph2), and SIFT (http://sift.jcvi.org) to assess any potentially damaging effect. Variants passing these filtering steps were considered to be most likely disease-causing and forwarded to validation process by Sanger sequencing. Additionally, genes with at least two heterozygous changes in the DNA sequence were considered to be most likely disease-causing, even though homozygous variants were not completely withdrawn.

2.4. Sanger Sequencing. We validated the candidate variations by Sanger sequencing in the two affected individuals and their parents. PCR primers were designed with Primer3 tool (http://frodo.wi.mit.edu/primer3/) to contain the mutation sites and their flanking regions (PCR primers and PCR reaction conditions are available upon request). PCR amplifications were inspected for single band of expected sizes on agarose gels before purification with Agencourt AMPure on Biomek NX (Beckman Coulter, USA). Sequencing was achieved using the automated ABI Prism 3730xlDNA Sequencer in combination with the Big Dye Terminator Cycle Sequencing Ready Reaction Kit 3.1 (Applied Biosystems, USA), and purification of sequencing reaction was performed with Agencourt CleanSEQ on Biomek NX(Beckman Coulter, USA). Sequences were assembled and analyzed with Mutation Surveyor software (SoftGenetics, USA).

\section{Results}

Exon-wide sequencing revealed two heterozygous mutations in exon 11 and exon 27, respectively, of TRAPPC11 in both affected children. Both of them were single base substitution c.1192C>T (p.Arg398*) and c.3014C>T (p.Pro1005Leu). Sanger sequencing confirmed the two heterozygous mutations in the patients and each was inherited from one of the parents (c.1192C>T (p.Arg398*) from the mother and c.3014C $>$ T (p.Pro1005Leu) from the father (Figure 1). The two mutations were found to be conserved across multiple species and in known functional domains of the TRAPPC11 gene (Figure 2).

So far, there were only four mutations in TRAPPC11 reported in patients. The two mutations detected in our patients have not been described in patients. The c.1192C > T (p.Arg398*) was observed as rs140403642 in the NHLBI GO Exome Sequencing Project database (ESP, http://evs.gs.washington.edu/EVS/) with a MAF of 0.000077 , c.3014C $>$ T (p.Pro1005Leu). The variants were found as in the ExAC database in 1 and 5 samples, corresponding to a frequency of $8.2 \mathrm{e}-06$ and $4.1 \mathrm{e}-05$, respectively. In ExAC they were not detected in non-Caucasian ethnicity samples and thus represent novel mutations in Asian ethnicity. Likewise, they were not found in the 1000 genomes database and they were undescribed in the ClinVar database.

\section{Discussion}

In this report, we identified by whole-exome sequencing a compound heterozygous mutations in TRAPPC11 (AK022778.1), i.e., c.1192C>T (p.Arg398*) and c.3014C>T (p.Pro1005Leu), in two affected members of a Chinese family with LGMD. Previous findings include the wholeexome sequencing combined with linkage analysis of a 
(A)

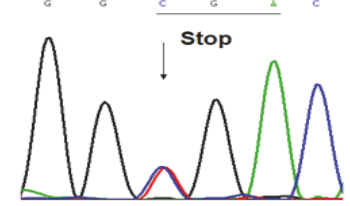

(B)

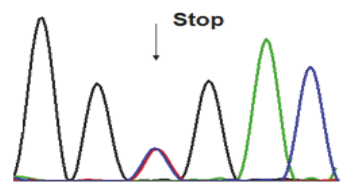

I

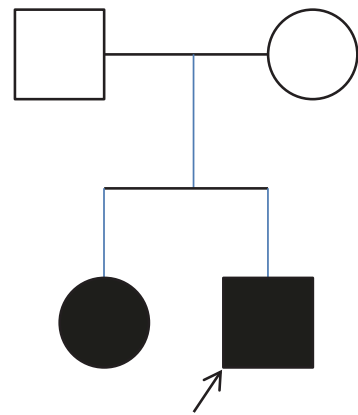

(a)
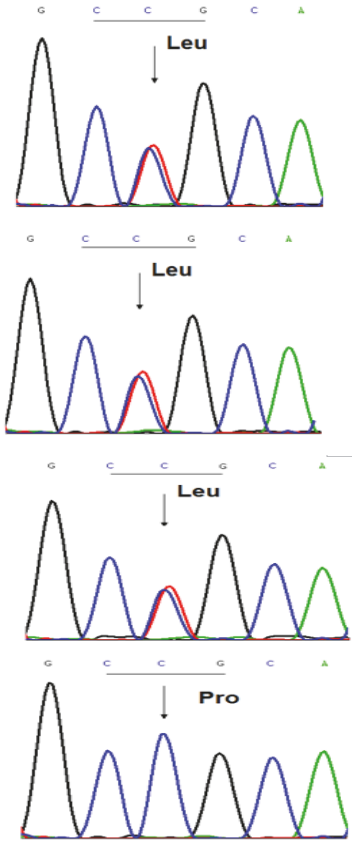

(b)

FIgURE 1: (a) Pedigree of family and (b) Sanger sequencing results from TRAPPC11 mutations. The left column shows the c.1192C $>$ T (p.Arg398*) mutation and the right column shows the c.3014C > T (p.Pro1005Leu) mutation. The two firsts row ((A) and (B)) shows results from the children. The next rows $((C)$ and $(D))$ show the results from the parents.

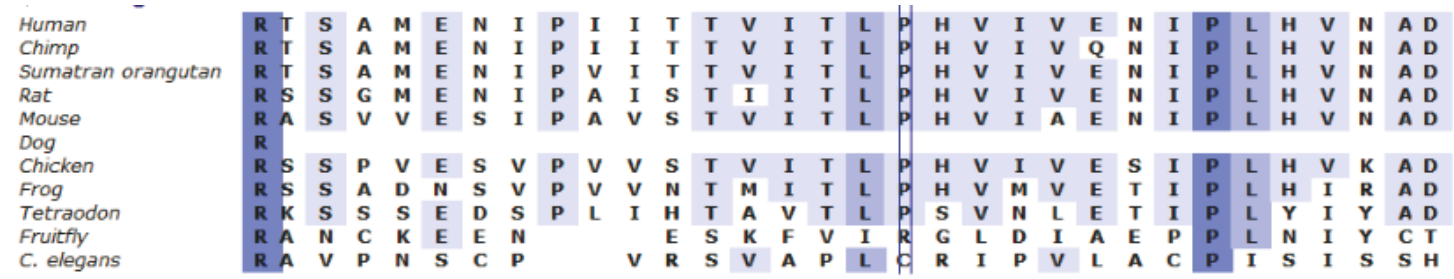

(a)

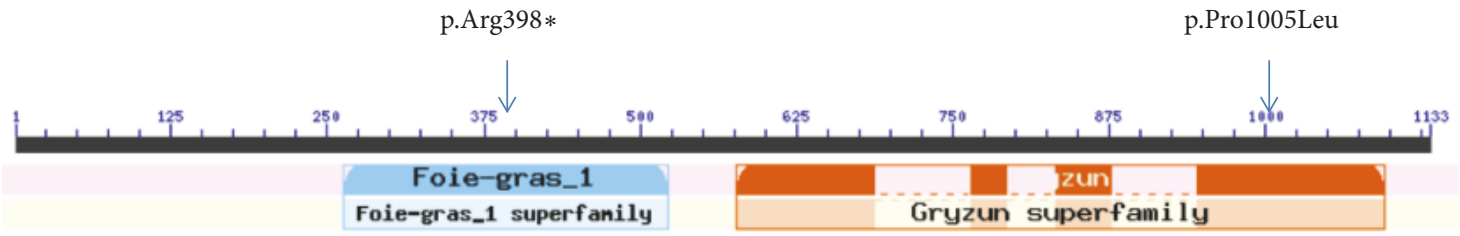

(b)

FIgURE 2: (a) Alignment of the c.1192C > T (p.Arg398*) mutation across species, illustrating a conserved region. (b) Indication of both c.1192C > T (p.Arg398*) and c.3014C > T (p.Pro1005Leu) mutations and their position in relation to functional domains of the gene.

Syrian family with limb girdle muscular dystrophy type 2S (LGMD2S;OMIM\#615356). Further, Bogershausen et al. identified a homozygous mutation in the TRAPPC11 gene (G980R) [13]. The authors also detected a different homozygous mutation in the TRAPPC11 gene (Ala372_Ser429del) in affected members of 2 Hutterite families with a slightly different phenotype. The G980R mutation occurred in the gryzun domain, whereas the deletion occurred in the foie gras domain. Similarly, c.1192C>T (p.Arg398*) mutation and c.3014C $>\mathrm{T}$ (p.Pro1005Leu) mutation reported in this study also occurred in the foie gras domain and the gryzun domain, respectively (Figure 2). Recent additional work further expands the breadth of findings, as summarized in Table 1 [14-16].

The affected individuals with TRAPPC11 mutations were described with two groups of clinical manifestations: one with more prominent muscular and skeletal symptoms and the other with microcephaly, hyperkinetic movements, 


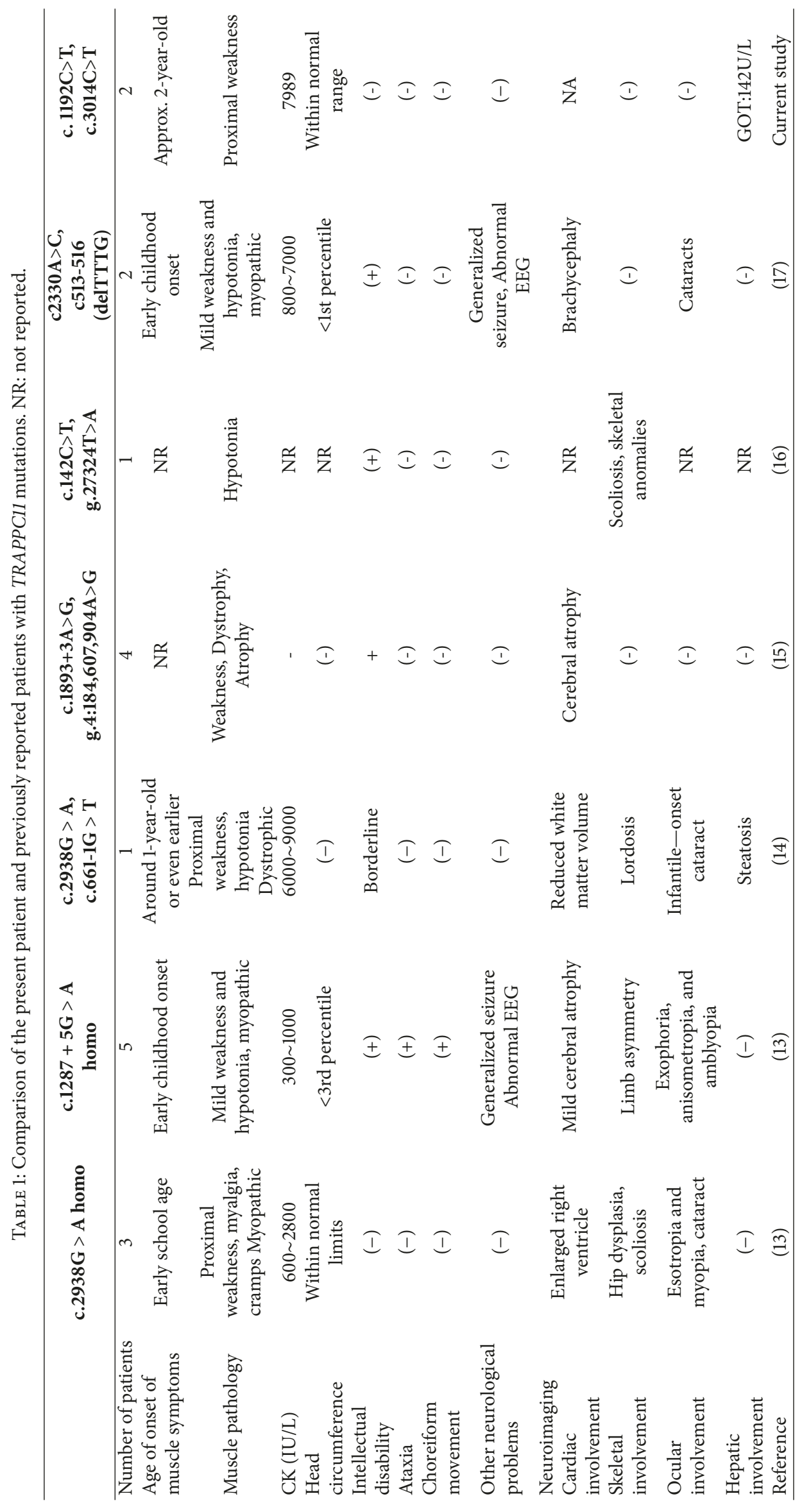


ataxia, and intellectual disability, which was discussed as a reflection of the difference of the two genotypes, Gly980Arg and Ala372_Ser429del [13]. Most recently, Liang et al. reported a Chinese girl harboring a compound heterozygous c.2938G > A/c.661-1G > T mutations in TRAPPC11 presenting congenital muscular dystrophy, fatty liver, and infantileonset cataract, demonstrating the broad spectrum of disease phenotypes arising from TRAPPC11 mutation in human [17]. The affected individuals from the Chinese family of LGMD2S reported herein were mainly characterized with progressive proximal muscle weakness resulting in impaired ambulation, difficulty to climb stairs, and increased serum creatine kinase. We did not observe noticeable features of nervous and hepatic involvement in the two patients with LGMD2S. The results clearly revealed that there is significant variability in phenotypes of TRAPPC11 mutations.

TRAPPC11 is a component of the TRAPP multisubunit tethering complex involved in intracellular vesicle trafficking [18]. Patient cells from both groups showed increased fragmentation of the Golgi apparatus and decreased amounts of the mutant proteins. Studies in yeast suggested that the mutant missense protein lost the ability to interact properly with other TRAPP proteins. Patient cells also showed altered protein transport along the secretory pathway, with a delayed exit from the Golgi and a defect in the formation and/or movement of late endosomes/lysosomes [13]. Liang et al. did not observe full-length TRAPPC11 protein in the patient harboring c.2938G > A/c.661-1G > T compound heterozygous mutations [17]. The findings suggested that altered membrane trafficking is the underlying molecular mechanism of this disease spectrum. Interestingly TRAPPC11 has also been associated with glycosylation [19] and later studies on zebra fish have confirmed that TRAPPC11 is involved in protein glycosylation [20], providing for a more detailed hypothesis on disease mechanism in this case report.

In conclusion, this study widens the phenotype of TRAPPC11 mutation related disorder and provides a conclusive case report for LGMD.

\section{Conflicts of Interest}

The authors declare that they have no conflicts of interest.

\section{Acknowledgments}

The authors wish to thank the patients and their parents who participated in this study. This work was supported by Science and Technology Project of Guizhou Province in China (no. [2016]7141), Science and Technology Innovation Talent Team Project of Guizhou Province (no. [2015]4019), and High-Level Innovative Talents Training Project of Guizhou Province (no. GZSYQCC[2016]004).

\section{References}

[1] X. Q. Rosales and C.-Y. Tsao, "Childhood onset of limb-girdle muscular dystrophy," Pediatric Neurology, vol. 46, no. 1, pp. 1323, 2012.
[2] K. Bushby, "Diagnosis and management of the limb girdle muscular dystrophies," Practical Neurology, vol. 9, no. 6, pp. 314323, 2009.

[3] E. Pegoraro and E. Hoffman, "Limb-Girdle Muscular Dystrophy Overview, In GeneReviews," 2002.

[4] R. A. Pagon, T. D. Bird, C. R. Dolan, and et al., Eds., University of Washington, Seattle, WA, USA, 1993, http://www.ncbi.nlm.nih .gov/books/NBK1408/.

[5] C. Minetti, F. Sotgia, C. Bruno et al., "Mutations in the caveolin3 gene cause autosomal dominant limb-girdle muscular dystrophy," Nature Genetics, vol. 18, no. 4, pp. 365-368, 1998.

[6] I. Richard, O. Broux, V. Allamand et al., "Mutations in the proteolytic enzyme calpain 3 cause limb-girdle muscular dystrophy type 2A," Cell, vol. 81, no. 1, pp. 27-40, 1995.

[7] E. Mercuri and F. Muntoni, "The ever-expanding spectrum of congenital muscular dystrophies," Annals of Neurology, vol. 72, no. 1, pp. 9-17, 2012.

[8] H. Li and R. Durbin, "Fast and accurate long-read alignment with Burrows-Wheeler transform," Bioinformatics, vol. 26, no. 5, pp. 589-595, 2010.

[9] A. McKenna, M. Hanna, E. Banks et al., "The genome analysis toolkit: a MapReduce framework for analyzing next-generation DNA sequencing data," Genome Research, vol. 20, no. 9, pp. 1297-1303, 2010.

[10] M. A. Depristo, E. Banks, R. Poplin et al., "A framework for variation discovery and genotyping using next-generation DNA sequencing data," Nature Genetics, vol. 43, no. 5, pp. 491-501, 2011.

[11] D. C. Koboldt, K. Chen, T. Wylie et al., "VarScan: Variant detection in massively parallel sequencing of individual and pooled samples," Bioinformatics, vol. 25, no. 17, pp. 2283-2285, 2009.

[12] K. Wang, M. Li, and H. Hakonarson, "ANNOVAR: functional annotation of genetic variants from high-throughput sequencing data," Nucleic Acids Research, vol. 38, no. 16, article e164, 2010.

[13] N. Bögershausen, N. Shahrzad, J. X. Chong et al., "Recessive TRAPPC11 mutations cause a disease spectrum of limb girdle muscular dystrophy and myopathy with movement disorder and intellectual disability," American Journal of Human Genetics, vol. 93, no. 1, pp. 181-190, 2013.

[14] K. Koehler, M. P. Milev, K. Prematilake et al., "A novel TRAPPC11 mutation in two turkish families associated with cerebral atrophy, global retardation, scoliosis, achalasia and alacrima," Journal of Medical Genetics, vol. 54, no. 3, pp. 176$185,2017$.

[15] M. K. Eldomery, Z. Coban-Akdemir, T. Harel et al., "Lessons learned from additional research analyses of unsolved clinical exome cases," Genome Medicine, vol. 9, no. 1, article no. 26, 2017.

[16] D. B. Fee, M. Harmelink, P. Monrad, and E. Pyzik, "Siblings with Mutations in TRAPPC11 Presenting with Limb-Girdle Muscular Dystrophy 2S," Journal of Clinical Neuromuscular Disease, vol. 19, no. 1, pp. 27-30, 2017.

[17] W. Liang, W. Zhu, S. Mitsuhashi et al., "Congenital muscular dystrophy with fatty liver and infantile-onset cataract caused by TRAPPC11 mutations: broadening of the phenotype," Skeletal Muscle, vol. 5, no. 1, 2015.

[18] P. J. Scrivens, B. Noueihed, N. Shahrzad, S. Hul, S. Brunet, and M. Sacher, "C4orf41 and TTC-15 are mammalian TRAPP components with a role at an early stage in ER-to-Golgi trafficking," Molecular Biology of the Cell (MBoC), vol. 22, no. 12, pp. 2083-2093, 2011. 
[19] L. Matalonga, M. Bravo, C. Serra-Peinado et al., "Mutations in TRAPPC11 are associated with a congenital disorder of glycosylation," Human Mutation, vol. 38, no. 2, pp. 148-151, 2017.

[20] C. DeRossi, A. Vacaru, R. Rafiq et al., "Trappc1l is required for protein glycosylation in zebrafish and humans," Molecular Biology of the Cell (MBoC), vol. 27, no. 8, pp. 1220-1234, 2016. 


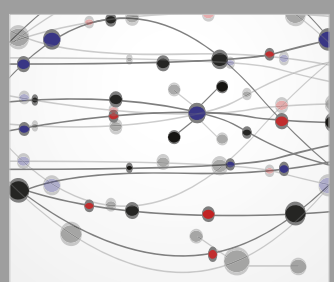

The Scientific World Journal
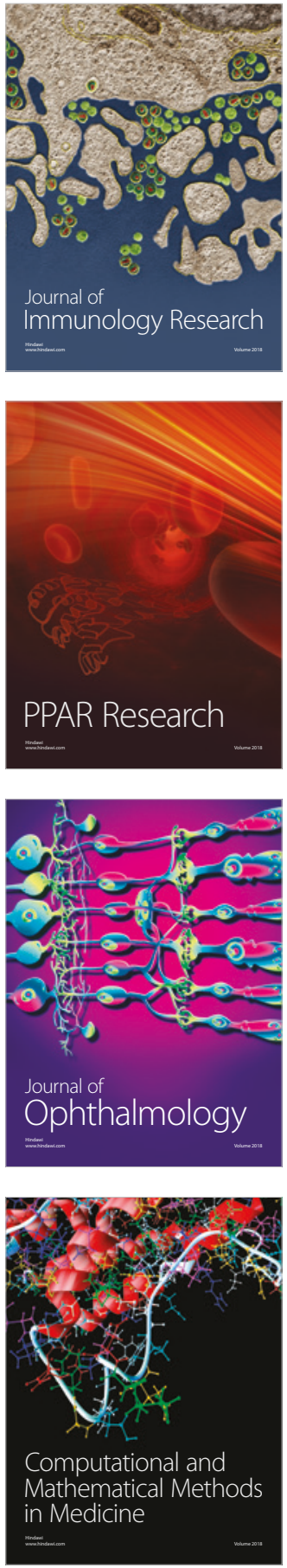

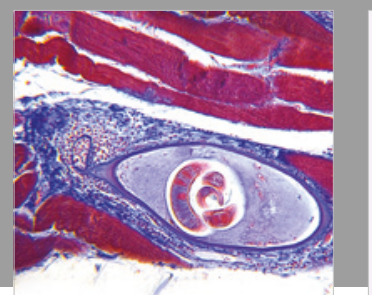

Gastroenterology Research and Practice

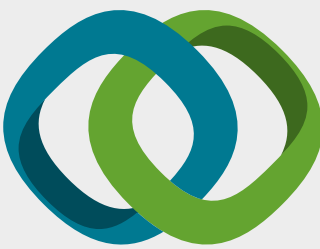

\section{Hindawi}

Submit your manuscripts at

www.hindawi.com
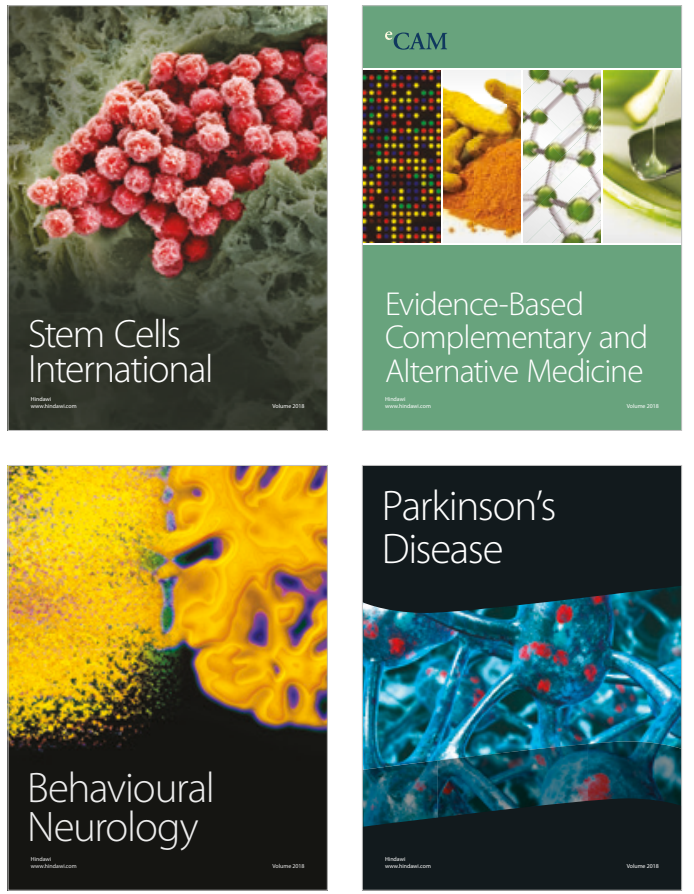

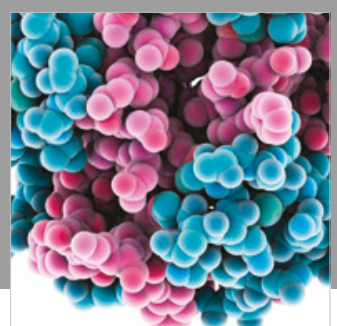

ournal of

Diabetes Research

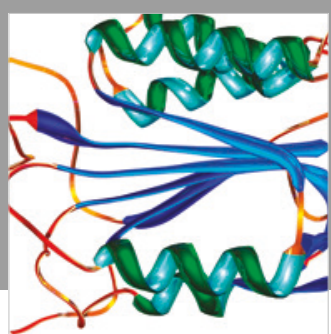

Disease Markers
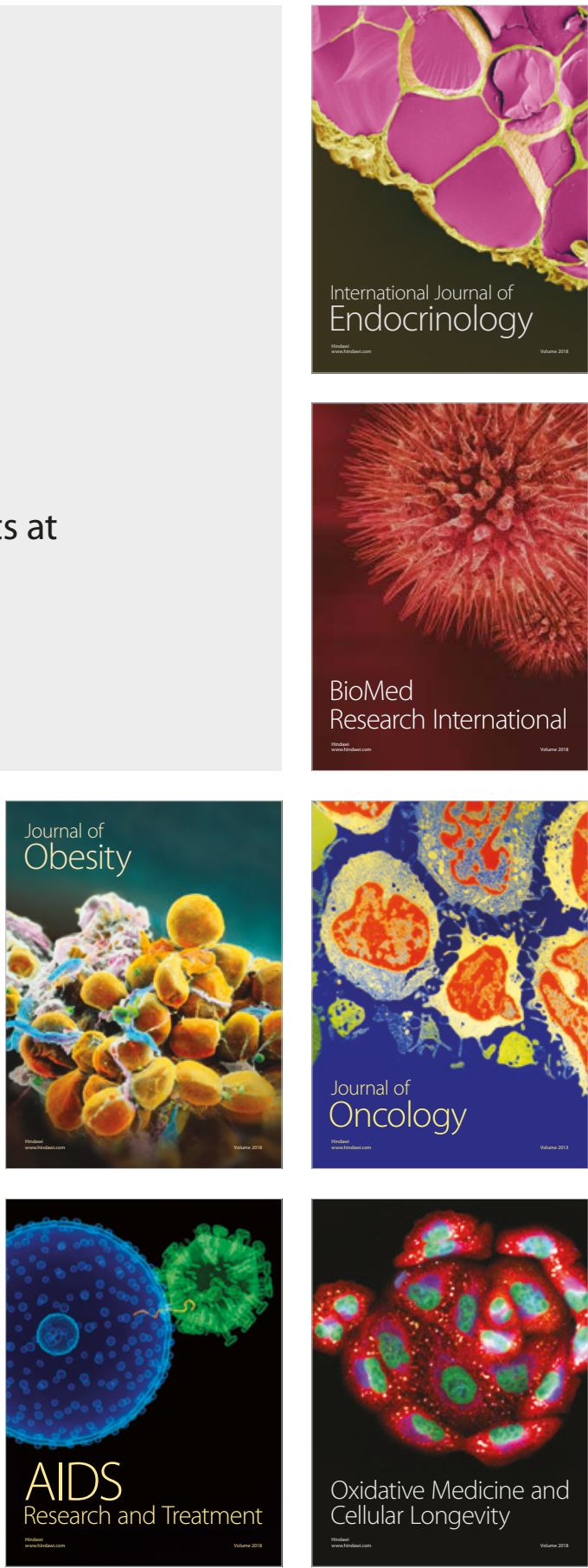